\title{
Metabolomics and its potential in diagnosis, prognosis and treatment of rheumatic diseases
}

\section{Żaneta Smoleńska, Zbigniew Zdrojewski}

Department of Internal Medicine, Connective Tissue Diseases, and Geriatrics, Medical University of Gdansk, Gdansk, Poland

\begin{abstract}
The main aim of metabolomics is to make a comprehensive study of metabolites, the intermediates of biochemical processes in living organisms. Any pathophysiological mechanism caused by disease will inevitably lead to related changes in the concentrations of specific metabolites. In line with this, metabolomics offers a promising laboratory tool for the analysis of potential diagnostic biomarkers that may be used to assess susceptibility to a disease and to evaluate the prognosis and therapeutic response to treatment.

Recent data have shown that metabolomics analysis in rheumatoid arthritis has made possible more efficient diagnosis, discrimination between patients with regard to disease activity, prediction of the response to a particular treatment approach, differentiation between rheumatic disease subtypes and greater understanding of the pathophysiology of this disease.

Here we characterize metabolomics as a comprehensive laboratory tool and review its potential in the diagnosis, prognosis and treatment of rheumatic diseases such as rheumatoid arthritis.
\end{abstract}

Key words: rheumatoid arthritis, biomarkers, metabolomics, early rheumatoid arthritis.

\section{Introduction}

Metabolomics complements genomics, transcriptomics and proteomics in the comprehensive analysis of biological systems in health and disease. Recent developments in analytical technology have turned it into an extremely powerful laboratory methodology that has increasingly been used in biomedical and clinical research [1]. The main aim of metabolomics is the comprehensive study of metabolites, the intermediates of the biochemical processes that occur in living organisms. Specifying which metabolites can be measured by means of metabolomics depends on the biological sample and the laboratory technique employed. They are usually defined as any molecules of a molecular weight of less than 1000 daltons. It has been suggested that the definition of metabolites should also include all small molecules introduced and modified by diet, medication, environmental exposure and coexisting organisms [2]. From a diagnos- tic and clinical point of view, the following advantages of a study of metabolites should be stressed:

- the short rapid response of the metabolic pattern to any physiological change in the organism,

- the association between the functions of specific genes,

- the impact of the metabolite pattern on the activity of proteins and genes,

- the straightforward relationship between metabolite concentration and disease activity.

Metabolomics enables concentrations of a wide range of components to be measured in a single sample [2]. Any pathophysiological mechanism caused by a disease in a biological system will inevitably lead to related changes in the concentrations of specific metabolites. In line with this, metabolomics allows the specific responses of organisms to environmental stimuli to be broadly analyzed, a procedure that is gaining popularity in drug research and clinical studies. 
From the pharmacological point of view, this methodology makes it possible to perform a rapid and comprehensive assessment of complex biological responses to new chemical entities and the effects of external stimuli, such as pharmaceutical dosages [1]. The importance of metabolomics for the study of disease biomarkers has been recently acknowledged $[3,4]$.

\section{Approaches to the study of metabolomics}

The analysis of a metabolome can be approached in three main ways:

1) fingerprinting,

2) non-targeted analysis,

3) targeted analysis $[2,5,6]$.

Metabolomic fingerprinting is applied to sample sets in which initial differentiation is performed on the basis of an unbiased, detailed and reproducible analytical fingerprint that represents many of the diverse compound classes of metabolomes. Non-targeted metabolomics as a method of metabolic profiling makes possible the detection and analysis of the complete metabolome or panels such as lipids, including phospholipids, amino compounds, sugars and bile acids, without focusing on a specific compound $[2,7,8]$ as targeted metabolomics does, measuring analytes that have been selected a priori on the basis of known biochemistry and/or previously established non-targeted studies. With the use of highly qualified statistical analysis, the changes in the metabolites can be mapped to specific pathways, enabling information to be extracted on the whole pathophysiological process of a particular disease. Metabolomics may offer the opportunity to map patients according to the specific pathways affected by their pathologies.

\section{Techniques for studying metabolomics}

Wide-ranging techniques such as nuclear magnetic resonance (NMR) or mass spectrometry (MS) have made metabolomics a highly relevant comprehensive tool that allows patterns of changes in biological systems to be described in order to understand better a particular disease pathway $[1,9]$. Nuclear magnetic resonance and MS can be used to acquire experimental information of biological systems including analysis of available biological materials such as blood, fluids and tissues. Among the benefits of these techniques are that they both require a minimal amount of sample (less than $1 \mathrm{ml}$ for liquids and $1 \mathrm{mg}$ for solids) and offer a spectroscopic pattern of tens or hundreds of measured metabolites. There are, however, several differences between the two techniques. Nuclear magnetic resonance has the advantage of better reproducibility, does not require sample pretreatment and is non-destructive, allowing multiple analyses of the same sample. Mass spectrometry has a much greater sensitivity but requires sample pretreatment and has to be linked to chromatography to allow separation into the different class of components. Mass spectrometry technique is more platform-dependent and susceptible to variability [10].

After obtaining the data, comprehensive statistical tools and models are used to extract the metabolomic information and to identify the metabolites and their correlations in order to differentiate between various pathologies, to determine the progression of disease and to discover potential biomarkers.

\section{Metabolomics in clinical studies}

Metabolomics has been applied to the study of several diseases as a potential tool for understanding the disease mechanisms and a relevant instrument in diagnosis and in the monitoring of treatment [11]. It has been shown that metabolomics analysis is potentially of discriminative value in the prognosis and diagnosis of diseases such as coronary heart disease and different degrees of stenosis [12], ocular inflammatory diseases [11], inflammatory bowel disease [13] and cancer [14]. It has been indicated that the nutritional status of rheumatoid arthritis patients is determined by the intensity of the chronic inflammatory process observed and by disease duration [15], which can be reflected in changes in some metabolites.

In recent years, the identification of biomarkers has been recognized as a challenging approach in many fields of medicine, including rheumatology. According to the working group of the National Institutes of Health for the U.S.A, a biomarker is considered "a characteristic that is objectively measured and evaluated as an indicator of normal biologic processes, pathogenic processes, or pharmacologic responses to a therapeutic intervention" [16]. Biomarkers play a role in diagnosis of disease with regard both to susceptibility and to evaluation of prognosis and therapeutic response.

Identification of potential biomarker candidates is a required step not only for diagnosis but also for better understanding of pathophysiology of a disease and in particular of its functional metabolism.

\section{Metabolomics as a potential tool for biomarker studies in rheumatic diseases}

The identification of patients who are susceptible to developing rheumatoid arthritis (RA), the most severe and persistent form of rheumatic disease, is a challenge for clinicians and researchers alike. Rheumatoid arthritis is a chronic inflammatory disease with joint involvement and systemic inflammation characterized by profound 
changes in the immune system. Early diagnosis and differentiation between early RA and other rheumatic diseases at the early stage presents major difficulties for rheumatologists, as there is a pressing need to be able to identify those patients with inflammatory arthritis who are at high risk of developing persistent and erosive disease [17]. Many studies have shown the effectiveness of disease-modifying anti-rheumatic drugs (DMARDs) in the treatment of patients with early RA before the first radiographic evidence of erosions, thus preventing further joint damage and disability. In line with this, the other important aspect of the study of rheumatic diseases is the creation of a new approach which enables DMARDs to be administered at a very early stage of RA [18] and which allows this treatment to be monitored.

Recent data have shown that metabolomic analysis in clinical RA studies has improved disease diagnosis, differentiation between disease subtypes and understanding of metabolic activity and the effect of drug treatment on metabolic profile. The results of the findings are presented in Tables I and II.

Elevated lipoprotein, a cholesterol-rich protein/lipid fraction similar to low density lipoprotein, has been noted in RA and constitutes an important cause of cardiovascular disease in RA. Lipid profile was analyzed in synovial fluid obtained from RA patients, and 70 different lipid components were identified. Among these, considerable amounts of 5S,12S-dihydroxyeicosatetraenoic acid (5S,12S-diHETE), an isomer of the pro-resolving mediator LTB4 with anti-inflammatory properties, were identified [19].
A potential application of metabolomic methodology for RA was emphasized in a study that demonstrated that some endogenous metabolites may discriminate patients with regard to different disease activity [20] or even predict the response to a particular treatment [21]. In particular, metabolic changes were indicated related to the response to methotrexate (MTX) treatment taken as monotherapy in patients with early RA. It was also shown that patients with active RA after responding to MTX exhibited elevated uric acid, taurine, methionine, glycine, histidine and hypoxanthine serum levels while patients for whom MTX was not effective showed decreased uracil, trimethylamine n-oxide (TMAO), oxoglutarate, aspartate, and tryptophan serum levels. The authors of the study suggested that these endogenous metabolites could be used to predict the therapeutic effect of MTX in RA patients [21].

Metabolomics has been tested in its capacity to differentiate RA from other diseases, including psoriatic arthritis (PsA) and osteoarthritis (OA) [22]. Metabolomics allowed patients with RA or PsA with predominant peripheral arthritis to be distinguished with a sensitivity of $90 \%$ and a specificity of $94 \%$. In particular, the study indicated that glutamine, heptanoic acid, succinate, pseudouridine, inosine, guanosine, arabitol, cystine, cysteine and phosphoric acid were increased in RA patients compared to PsA patients, whereas aspartic and glutamic acid, histidine, serine, arachidonic acid, cholesterol, threonic acid and 1-monooleoylglycerol were all decreased compared with PSA patients.

Table I. Metabolomic changes in rheumatoid arthritis - potential biomarkers predicting therapeutic effect

\begin{tabular}{|ll|}
\hline Metabolites that could be used to predict therapeutic effect & \multicolumn{1}{c|}{ Changes that have been indicated } \\
\hline $\begin{array}{l}\text { Uric acid, taurine, methionine, glycine, histidine, hypoxan- } \\
\text { thine }\end{array}$ & $\begin{array}{l}\text { Elevated levels after responding to MTX with respect to pa- } \\
\text { tients for whom MTX was not effective [21] }\end{array}$ \\
\hline Uracil, TMAO, oxoglutarate, aspartate, tryptophan & $\begin{array}{l}\text { Decreased levels after responding to MTX with respect to } \\
\text { patients for whom MTX was not effective [21] }\end{array}$ \\
\hline
\end{tabular}

Table II. Metabolomic changes in rheumatoid arthritis - potential diagnostic biomarkers

\begin{tabular}{|ll|}
\hline \multicolumn{2}{|c|}{$\begin{array}{c}\text { Metabolites that could be used as potential diagnostic } \\
\text { biomarkers }\end{array}$} \\
\hline $\begin{array}{l}\text { Glutamine, heptanoic acid, succinate, pseudouridine, ino- } \\
\text { sine, guanosine, arabitol, cystine, cysteine and phosphoric } \\
\text { acid }\end{array}$ & Increased levels in RA patients compared to PsA patients [20] \\
\hline $\begin{array}{l}\text { Aspartic and glutamic acid, glutamate, histidine, serine, ara- } \\
\text { chidonic acid, cholesterol, threonic acid, 1-monooleoylglycerol }\end{array}$ & Decreased levels in RA compared with PsA patients [20] \\
\hline $\begin{array}{l}\text { Succinate, octadecanol, asparagine, terephthalate, salicy- } \\
\text { laldehyde, glutamine, citrulline, tyrosine, uracil, lysine, ribi- } \\
\text { tol, tryptophan, xylose and ribose }\end{array}$ & Higher levels in the RA group than the non-RA group [3, 19] \\
\hline $\begin{array}{l}\text { Isopalmitic acid, glycerol, myristic acid, palmitoleic acid, hy- } \\
\text { droxylamine and ethanolamine }\end{array}$ & Lower level in the RA group than in the non-RA group [3, 19] \\
\hline
\end{tabular}


Using a metabolomics approach, Kim et al., in a study of 38 synovial fluid samples of inflammatory arthritis including RA, observed changes in the 20 metabolites selected as potential biomarkers to discriminate RA from non-RA patients [3]. As many as 105 metabolites were identified and classified into the following chemical classes: sugars and sugar alcohols, amino acids, fatty acids, organic acids, amines, phosphates and miscellaneous. The metabolite profiles of synovial fluid obtained from RA patients were distinguishable from those of other inflammatory arthritis. Finally 20 metabolites were selected and validated as potential biomarkers with the capability of discriminating RA from non-RA diseases with $92.3 \%$ sensitivity and $68.0 \%$ specificity [3]. The study indicated that succinate, octadecanol, asparagine, terephthalate, salicylaldehyde, glutamine, citrulline, tyrosine, uracil, lysine, ribitol, tryptophan, xylose and ribose were higher in the RA group than in the nonRA group. The level of isopalmitic acid, glycerol, myristic acid, palmitoleic acid, hydroxylamine and ethanolamine were lower in the RA group than in the non-RA group [3].

These metabolites are major intermediates of various metabolic pathways, including glycolysis, the tricarboxylic acid (TCA) cycle and pathways involving amino acid and fatty acid metabolism. In the TCA cycle alpha-ketoglutarate is a precursor to such amino acids as glutamate, glutamine, proline and arginine. Oxaloacetate, which is converted from succinate, fumarate and malate, is also a precursor to such amino acids as asparagine, methionine, threonine, isoleucine and lysine [22]. Citrulline synthesized from ornithine and carbamoyl phosphate is a key intermediate of the urea cycle. It is also generated through post-translational modification of arginine residues by peptidylarginine deiminase [20]. Citrulline is known as a major antigenic determinant recognized by RA. Anti-cyclic citrullinated peptide antibodies (anti-CCP) have been established as a useful tool to discriminate RA from other arthritic diseases [21].

The studies showed that the urea and TCA cycles as well as amino acid metabolism were highly activated in the RA group compared with the non-RA group. According to the results, citrulline, succinate, asparagine, glutamine and lysine are proposed as major biomarkers for RA diagnosis.

\section{Summary}

With regard to rheumatic diseases, the outcome of a particular disease is often strictly dependent on early diagnosis and the ability to predict a therapeutic response. Metabolomics has been considered a novel and promising tool in the clinical approach to rheumatic disease. The great advantages of this method include the possibility of producing complex metabolic profiles of patients with a very small amount of any available biological material as a starting point. In this respect, metabolomics enables studies of substances that may turn out to be candidate biomarkers.

The authors declare no conflict of interest.

\section{References}

1. Rajamäki TJ, Jämsen E, Puolakka PA, et al. Metabolomics in rheumatic diseases: The potential of an emerging methodology for improved patient diagnosis, prognosis, and treatment efficacy. Autoimmun Rev 2013; 12: 1022-1030.

2. Drexler DM, Reily MD, Shipkova PA. Advances in mass spectrometry applied to pharmaceutical metabolomics. Anal Bioanal Chem 2011; 399: 2645-2653.

3. Kim S, Hwang J, Xuan J, et al. Global metabolite profiling of synovial fluid for the specific diagnosis of rheumatoid arthritis from other inflammatory arthritis. PLoS One 2014; 9: 9.

4. Mamas M, Dunn WB, Neyses L, Goodacre R. The role of metabolites and metabolomics in clinically applicable biomarkers of disease. Arch Toxicol 2011; 85: 5-17.

5. Sangster T, Major H, Plumb R, et al. A pragmatic and readily implemented quality control strategy for HPLC-MS and GCMS-based metabonomic analysis. Analyst 2006; 131: 10751078.

6. Sumner LW, Amberg A, Barrett D, et al. Proposed minimum reporting standards for chemical analysis. Metabolomics 2007; 3: 211-221.

7. Paik MJ, Moon SM, Kim KR, et al. Target metabolic profiling analysis of free amino acids in plasma as EOC/TBDMS derivatives by GC-SIM-MS. Biomed Chromatogr 2008; 22: 339-342.

8. Wei R, Li G, Seymour AB. High-throughput and multiplexed LC/MS/MRM method for targeted metabolomics. Anal Chem 2010; 82: 5527-5533.

9. Trygg J, Holmes E, Lundstedt T. Chemometrics in metabonomics. J Proteome Res 2007; 6: 469-479.

10. Nicholson JK, Holmes E, Kinross JM, et al. Metabolic phenotyping in clinical and surgical environments. Nature 2012; 491: 384-392.

11. Young SP, Nessim M, Falciani F, et al. Metabolomic analysis of human vitreous humor differentiates ocular inflammatory disease. Mol Vis 2009; 15: 1210-1217.

12. Brindle JT, Antti H, Holmes E, et al. Rapid and noninvasive diagnosis of the presence and severity of coronary heart disease using 1H-NMR-based metabolomics. Nat Med 2002; 8: 1439-1444.

13. Lin HM, Helsby NA, Rowan DD, Ferguson LR. Using metabolomic analysis to understand inflammatory bowel diseases. Inflamm Bowel Dis 2011; 17: 1021-1029.

14. Ippolito JE, XU J, Jain S, et al. An integrated functional genomics and metabolomics approach for defining poor prognosis in human neuroendocrine cancers. Proc Natl Acad Sci U S A 2005; 102: 9901-9906.

15. Targonska-Stepniak B, Majdan M. Associations between parameters of nutritional status and disease activity in patients 
with rheumatoid arthritis. Pol Arch Med Wewn 2011; 121: 122-127.

16. Jenkins M, Flynn A, Smart T, et al. A statistician's perspective on biomarkers in drug development. Pharm Stat 2011; 10 494-507.

17. Bijlsma JWJ. Optimal treatment of rheumatoid arthritis: EULAR recommendations for clinical practice. Pol Arch Med Wewn 2010; 120: 347-353.

18. Semenova O, Thompson H, Kallankara S, et al. Treat to target in early rheumatoid arthritis clinic (EAC). Low radiological progression and good functional outcomes on conventional disease modyfying drugs (DMARDS). Ann Rheum Dis 2013; 72 (suppl. 3): A250-A257.

19. Giera M, loan-Facsinay A, Toes R, et al. Lipid and lipid mediator profiling of human synovial fluid in rheumatoid arthritis patients by means of LC-MS/MS. Biochim Biophys Acta 2012; 1821: 1415-1424.

20. Lauridsen MB, Bliddal H, Christensen R, et al. H-1 NMR spectroscopy-based interventional metabolic phenotyping: a cohort study of rheumatoid arthritis patients. J Proteome Res 2010; 9: 4545-4553.

21. Wang Z, Chen Z, Yang S, et al. H-1 NMR-based metabolomic analysis for identifying serum biomarkers to evaluate methotrexate treatment in patients with early rheumatoid arthritis. Exp Ther Med 2012; 4: 165-171.

22. Madsen RK, Lundstedt T, Gabrielsson J, et al. Diagnostic properties of metabolic perturbations in rheumatoid arthritis. Arthritis Res Ther 2011; 13: R19. 